\title{
Visión Formativa de la Metodología Cuantitativa en Educación
}

\author{
Autora: Neolany María Prieto Rivero \\ Universidad Fermín Toro, UFT \\ neolany.prieto@gmail.com \\ Lara, Venezuela
}

\section{Resumen}

La intencionalidad del presente ensayo, es precisar implicaciones epistémicas concernientes a una visión formativa de la metodología cuantitativa en el contexto de las ciencias educativas. En él, se ofrece un análisis que considera los elementos que integran su caracterización, tales como naturaleza, tipo y diseño de investigación, hipótesis, sistema de variable, técnicas e instrumentos de recolección de datos, validez y confiabilidad de un estudio orientado en dicho enfoque. Para ello, realicé una revisión bibliográfica para fundamentar su desarrollo y explicar de manera figurativa cada uno de los aspectos antes mencionados. Dicho enfoque, aplicado a una investigación científica refiere los procedimientos a seguir para dar respuesta a un problema a través de los objetivos planteados, en consonancia con la concepción ontológica, epistemológica y praxeológica de las prácticas sociales-discursivas de la actualidad. Todo ello, me permitió concluir que es tan importante incorporar todos los elementos que requiere el marco metodológico de toda investigación, como la pertinencia de la elección de todos y cada uno de ellos, atendiendo a la coherencia exigida en el paradigma asumido. En consecuencia, se debe ser cuidadoso para no entrar en contrariedades ni arbitrariedades que entorpezcan la culminación del trabajo, al confundir los términos empleados en el mismo.

Palabras clave: visión; metodología; educación. 


\title{
Formative Vision of the Quantitative Methodology in Education
}

\begin{abstract}
The intentionality of this essay is to specify epistemic implications concerning a formative vision of quantitative methodology in the context of the educational sciences. It provides an analysis that considers the elements that integrate its characterization, such as nature, type and design of research, hypotheses, variable system, techniques and instruments of data collection, validity and reliability of a study oriented in this approach. For this, it was made a bibliographical review to base its development and to explain in a figurative way each of the aspects mentioned above. This approach, applied to scientific research, refers to the procedures to be followed in order to respond to a problem through the stated objectives, in line with the ontological, epistemological and praxeological conception of current social-discursive practices. All of this allowed to conclude that it is so important to incorporate all the elements required by the methodological framework of all research, as well as the pertinence of the choice of each and every one of them, taking into account the coherence required in the assumed paradigm. Consequently, one must be careful not to enter into contradictions or arbitrariness that obstruct the culmination of work, by confusing the terms used in it.
\end{abstract}

Keywords: vision; methodology; education. 


\section{Introducción}

La investigación en ciencias de la educación constituye quizás una de las opciones de mayor relevancia que le da respetabilidad y legitimidad al proceso educativo. A través de ella, se promueven las competencias investigativas en todos los niveles, con el fin de lograr generar el conocimiento científico y tecnológico que necesitamos para una menor dependencia de los países que lo han logrado.

Al respecto, la aplicación de la metodología cuantitativa es la inquietud por desarrollar en este ensayo cuyo objetivo es dar una reseña sobre la sistematización utilizada en este tipo de investigación en el contexto de las Ciencias de la Educación. En él se plantea brevemente la epistemología del paradigma, el enfoque y sus métodos, todo ello con miras a señalar las diferentes estrategias que tenemos a mano y que se pueden usar en el campo de la educación con el objeto de dar solución a problemas que aquejan en dicho contexto.

Para plantear este enfoque, hay que reflexionar sobre el paradigma el cual constituye una visualización de una dificultad del objeto que se quiere conocer, cuya relación implica la utilización de métodos dirigidos a identificar, describir y analizar el problema, así como buscar su posible solución. Por ello, en la realización de este ensayo, producto de una revisión documental, se tomó en cuenta además el contenido del tema, la presentación, organización, ortografía, redacción, introducción de la idea, desarrollo y el cierre del mismo.

\section{Desarrollo}

El progreso del ser humano se basa en el desarrollo de la ciencia y tecnología, sustentado en la aplicabilidad de paradigmas en investigación que se llevan a cabo en las diferentes universidades donde se realizan trabajos de grado, fomentado el carácter innovador de la educación para el cambio, el cual se proyecta con una nueva posición de la filosofía universitaria. 
De esa manera, la universidad constituye un modelo de acción que promueve el encuentro con el conocimiento orientado bajo alguno de los paradigmas, lo que implica tomar en cuenta el contexto histórico que admite un modelo para conocer el sujeto consciente por el sujeto cognitivo y explicar bajo el enfoque metodológico del paradigma, los problemas vigentes que han estado presentes desde la modernidad hasta en la post modernidad en el seno de la sociedad a la cual se estudia.

Al respecto, en cada organización histórica social que el hombre como desarrollo humano genere, establece su propia razón, es decir, un modo de conceptualizar y hacer que le es específico, ésta es la llamada episteme, que de acuerdo con su raíz puede entenderse como conocimiento. La episteme según Moreno, (2008), es un modo de vida producido por un sistema de relaciones económicas, sociales y humanas, que abarca un período precisable en el tiempo, "se asemeja a una hechura particular del cerebro de los hombres en un momento de la historia. Es un modo general de conocer" (pág. 20).

Por consiguiente, las respuestas que el hombre busca están condicionadas por el conocimiento que tiene, en otras palabras, la episteme que viene a ser el camino para seguir en todo proceso o acto de conocimiento, aunque su régimen es duro y rígido, es flexible en la multiplicidad de formas en que se manifiestan los procesos cognoscitivos. Esto es lo que permite el surgimiento de los paradigmas, que viene a ser las distintas formas en que se despliega la episteme.

Mediante el establecimiento de un paradigma, se comparte el consenso, filosofías, teorías y métodos convenientes para un proceso de investigación; el mismo ha venido ocupando cada vez mayor espacio en el campo del conocimiento. Es el marco de pensamiento o referencia que orienta las actividades y las reflexiones dentro de un área determinada del conocimiento. Es Kuhn (1981), quien se da la tarea de elaborar teóricamente la noción de paradigma, y lo reseña como todo lo que comparte una comunidad científica, 
o a la inversa una comunidad científica son las personas que comparten o practican un paradigma; con el sentido de examinar críticamente el cambio de las prácticas científicas a través del rol de las funciones cognoscitivas y de las influencias sociales o históricas.

Según el precitado autor, toda actividad científica que se realice en una época estará determinada por uno o varios paradigmas correspondientes a ella que condicionarán los temas, el modo de abordarlos, métodos y todo lo que se relacione con el conocimiento y la manera de conocer. Como se puede deducir de lo indicado, el análisis de la realidad implica un proceso metodológico que es necesario conocer.

Ahora bien, cada problema o estudio de investigación utiliza un proceso metodológico, y dentro de éste las técnicas o estrategias empíricas que se consideren más adecuada, según el modelo conceptual o paradigma escogido, bien sea implícita o abiertamente. Al respecto, Rivero (2008), afirma que la investigación desde el punto de vista de los profesionales de la educación, es vista primordialmente como una actividad de documentación que se realiza en cualquiera de las fuentes bibliográficas existentes 0 en el Internet con el fin de satisfacer una necesidad curricular inmediata; documentación que conlleva a la formación académica de quien investiga y cuyos resultados se traducen en su praxis diaria.

En conformidad con lo anterior, se puede deducir que investigación es sinónimo de consulta instantánea, de información motivada por adquirir un nivel académico y no por un proceso permanente de adquisición de información, de herramientas cognitivas aplicables al quehacer educativo para la resolución de dificultades concretas previamente diagnosticadas y racionalizadas. Desde esta perspectiva, el conocimiento, elemento esencial dentro del proceso de investigación, es concebido como una estructura estática que no se dinamiza y construye de manera contextualizada a partir de la praxis investigativa. 
Cabe acotar, que la mayor parte de las investigaciones a nivel nacional está centrada en las universidades y dicho proceso se lleva a cabo de manera progresiva, puesto que los estudiantes de postgrado se encuentran motivados a mejorar la calidad académica por la vía institucional, así como para crear nuevos conocimientos que son impartidos, divulgados 0 utilizados en la resolución de problemas de las propias instituciones o de otros sectores de la sociedad que lo requieran.

Ante esa situación, es evidente que en los estudios de maestría o doctorado existe el acto investigativo y que el mismo se lleva a cabo en cualquiera de los paradigmas presentes, modelo que va a orientar todo el proceso del trabajo, por lo que se debe adoptar la metodología pertinente que permitirá el continuo devenir que fluya y facilite el proceso, porque todo es inacabado y compartido. Igualmente, debe existir un acto creador donde se le dé a la investigación un matiz personalizado recreado por el autor.

Con base a ello y para fines de este ensayo, analizaré específicamente el modelo conceptual desde la perspectiva positivista, cuyo paradigma se acompaña de un enfoque metodológico cuantitativo, y parte de la presunción que es posible establecer las causas de los hechos. De igual manera, de un sistema de hipótesis a través del método hipotético-deductivo de teorías previamente seleccionadas para contrastarlas, con la finalidad de confirmarlas o desecharlas.

En ese sentido, no se puede hablar de paradigma sin partir del significado dado por Kuhn (1971), como el conjunto de experiencias "...basadas firmemente en una o más realizaciones científicas pasadas, ...que alguna comunidad científica particular reconoce, durante cierto tiempo, como fundamento para su práctica posterior..." (pág. 33), en las cuales según el autor antes mencionado "Los hombres cuya investigación se basa en paradigmas compartidos están sujetos a las mismas reglas y normas para la práctica científica" (pág. 34) 
Dicho paradigma de investigación es aplicado en las ciencias llamadas exactas o naturales e incluso en las sociales, como es el caso de la educación en todos los niveles y modalidades. Al referirme a éstas últimas, los paradigmas en ellas utilizados se impregnan de experiencias, valores y creencias para percibir la realidad como una manera de entender el mundo y el conocimiento. No obstante, el positivismo demanda la sistematización de una metodología que guiará el transitar de la investigación, cuya naturaleza responderá al mismo.

En este orden de ideas, el enfoque metodológico que corresponde a los trabajos de investigación, enmarcados en dicho paradigma, es el cuantitativo que según Hernández, Fernández y Baptista (2010a), se caracteriza por medir fenómenos, utilizar estadística, probar hipótesis y hacer análisis causa efecto. Por consiguiente, sigue un patrón lineal deductivo según el método científico que incluye desde la observación del problema, planteamiento del mismo, interrogantes, objetivos, diseño, técnica de recolección de datos, así como la elaboración instrumentos tipo cuestionarios, análisis de resultados y elaboración de conclusiones.

Dentro de esa metodología sistematizada, existen elementos ineludibles que deben desarrollarse, de los cuales entre otros tomaré para el desarrollo de este ensayo, la naturaleza, tipo y diseño de investigación cómo puntos neurálgicos que generalmente, son confundidos $u$ obviados, denominados por Ollarves (2016a), como triada metodológica, en el entendido que no son los únicos, pero fundamentales en todo proceso investigativo.

En ese sentido, la naturaleza de la investigación como primer elemento de la triada, se refiere al paradigma y enfoque asumido que, en este caso, es el positivista que según Ollarves (2016b), asienta sus bases teóricas para explicar los planos del conocimiento (ontológico, epistemológico y metodológico) con características muy particulares. Así se tiene que desde la perspectiva epistemológica el sujeto cognoscente se mantiene independiente 
del objeto de estudio aún en las investigaciones de corte social como las educativos. Ello se debe, de acuerdo con la autora antes mencionada porque su pensamiento se mantiene desprendido del objeto, de tal manera como estaría al estudiar una célula en el microscopio.

Desde el plano ontológico, Ollarves (2016c), considera que, desde allí se puede conocer el objeto de estudio tal y como es en la realidad aparte de la voluntad de las personas; con la premisa de que dicha realidad es estática y fragmentable. En cuanto al metodológico, asume un enfoque cuantitativo que de acuerdo con Hernández, Fernández y Baptista (2010b), permite “...controlar, predecir una realidad única, tangible y fraccionada, siguiendo leyes para establecer una separación entre el sujeto cognoscente y el objeto de estudio, lo cual garantiza la neutralidad ante las situaciones a estudiar" (pág. 105).

En cuanto al tipo de investigación, como segundo elemento de la triada, reporta la clase de estudio a llevar a cabo y da cuenta de la manera en que se va a desarrollar. Está regido por los objetivos planteados. El mismo, puede ser de campo utilizado para recabar los datos in situ; en otras palabras, permite recogerlos de primera fuente, mediante la aplicación de un instrumento a una población. Éste, se clasifica de acuerdo con el nivel de conocimiento del estudio y en concordancia con lo planteado por la Universidad Experimental Libertador (UPEL, 2008a), que establece que los mismos pueden ser exploratorios (superficial), descriptivos (intermedio), explicativos (profundo).

Otro tipo de investigación es la documental, referidas a revisiones críticas del estado del conocimiento, compilación de fuentes orales/escritas, tal como lo señala Ollarves (2016d). De igual manera están las desarrolladas bajo la modalidad proyectiva, divididas por la UPEL (2008b), en proyectos especiales cuyo fin es la elaboración y desarrollo de modelo operativo viable para solucionar tecnología, problemas, métodos procesos; y los proyectos 
factibles, destinados a creaciones tangibles para dar soluciones a problemas: libros, programas, planes, software, entre otros.

En lo que se refiere al diseño de la investigación, como último pero no menos importante elemento de la triada, es el plan o estrategia concebida para obtener la información que se desea en la investigación. Éste se divide en experimentales y no experimentales. En los experimentales se manipulan una o más variables como es el caso de las ciencias naturales y los no experimentales son aquellos donde no se manipulan las variables; su objetivo es únicamente observar los fenómenos, tal y como suceden en su contexto natural para analizarlos, según Hernández, Fernández y Baptista (2010c). Dentro de los experimentales se encuentran:

Los pre-experimentales: se administra un estímulo o tratamiento a un grupo, y después aplica una medición de una o más variables para observar cuál es el nivel del grupo en éstas. Los expo-facto o post-prueba: donde existe punto de referencia inicial para ver qué nivel tenía el grupo en la(s) variable (s) dependiente (s) antes del estímulo, hay seguimiento del grupo. No resulta conveniente para establecer causalidad porque no hay manipulación ni grupo de comparación. Los experimentales puros: que tienen grupos equivalentes de comparación y se manipula la variable independiente. Utilizan pruebas antes (no todos) y después (todos), para analizar la evolución de los grupos antes y después del tratamiento experimental para determinar los efectos. Los cuasi Experimentales que manipulan deliberadamente, al menos, una variable independiente para observar su efecto y relación con una o más variables dependientes.

Por su parte, las investigaciones no experimentales, se clasifican en: transeccionales o transversales que a su vez pueden dividirse en descriptivos, correlacionales (incidencia) y correlacional causal (causa-efecto). Los longitudinales que pueden ser de tendencia, evolución de grupo, panel. 
En cuanto a las hipótesis de una investigación, son declaraciones claras, precisas y específicas en torno al fenómeno a investigar; las más usadas en educación son la que se refieren a causa-efecto pues explican la relación entre dos variables, pueden ser alternativas, nulas o estadísticas. Por otro lado, tenemos las variables que según Ritchey (2001), son fenómenos medibles que varían (cambian) a través del tiempo o que difieren de un lugar a otro o de un individuo a otro, las mismas deben estar inmersas en el título de la investigación.

Otro aspecto importante dentro de las investigaciones con enfoque metodológico cuantitativo, es la técnica de recolección de datos o registro cuantitativos, que va a depender de la naturaleza de los datos a recoger. En los estudios educativos, generalmente se utiliza la encuesta que según Hurtado y Toro (2006), "la información se obtiene a través de preguntas a otras personas, en ella no se establece un diálogo con el entrevistado, el grado de interacción es menor" (pág. 449). Con dicha técnica se aplican instrumentos tipo cuestionarios (son los más utilizados y consisten en un conjunto de ítems presentados en forma de afirmaciones o juicio, ante los cuales se pide la reacción de los sujetos).

Al respecto, según los autores antes mencionados, un instrumento de recolección de datos utilizado en una investigación para que sea considerado adecuado, debe registrar datos observables que representen verdaderamente los conceptos o variables que el investigador tiene en mente; a esto se le llama validez, la misma se comprueba sometiendo el cuestionario a la técnica de juicio de expertos, los cuales son especialistas en el área temática. Existen diferentes tipos de validez, la externa, interna y conceptual.

Aunado a la validez, hay que comprobar la confiabilidad del instrumento, que de acuerdo con los precitados autores, es uno de los requisitos de la metodología cuantitativa, fundamentada en el grado de uniformidad con que los instrumentos de medición cumplen su finalidad. En otras palabras, es la 
capacidad que tiene un instrumento al ser aplicado una serie de veces a un grupo de sujetos y por diferentes investigaciones, obtiene los mismos resultados con un máximo de exactitud y credibilidad; para ello, se aplica el estadístico del Alpha de Cronbach o Kuder y Richardson según la escala, y en los estudios correlacionales Pearson, Kendall o Spearman.

Después que el investigador termina la recolección de datos a través de los instrumentos, procede a organizar la información a través de la tabulación y cuantificación de los datos obtenidos en cuadros de frecuencia y porcentajes; para luego reflejarlo en gráficos para proceder a realizar el análisis; lo que significa que debe descomponer, separar y tomar un todo y dividirlos en sus elementos constitutivos, todo lo cual le permitirá razonar, analizar, así como constatar con los referentes teóricos epistemológicos seleccionados, que le ayudarán a dar respuesta a los objetivos planteados en la investigación y lo conducirán exitosamente a la fase conclusiva, para poder hacer las respectivas recomendaciones y hacer la propuesta, según sea el caso.

\section{Conclusiones}

Todo proceso de investigación es una necesidad en los estudios de postgrado bien sea magister o doctorado, a fin de que el participante estudie el estado del arte, y con ello favorezca la profundización del conocimiento que la praxis social le demanda consiente de la importancia de la formación permanente que debe tener.

Al hacer la consulta documental, pude evidenciar que no existe un criterio único acerca del uso ni definición de los términos empleados, encontrándome con autores que le endosan la palabra diseño a los tipos y hasta a la naturaleza de la investigación. Situación que contribuye a confundir al revisar lo que han escrito los autores respecto al tema, sobre todo a las personas y/o estudiantes que se inician en los quehaceres investigativos. 
Sin embargo, desde las consideraciones antes explicadas, es significativo resaltar la importancia de no sólo de incorporar todos los elementos que requiere el marco metodológico para el éxito de una investigación, sino la pertinencia de la elección de todos y cada uno de ellos, atendiendo a la coherencia exigida en el paradigma asumido para el desarrollo de la misma. En consecuencia, se debe ser cuidadoso (a) para no entrar en contrariedades ni arbitrariedades que entorpezcan el éxito en la culminación del trabajo, al confundir los términos empleados en el mismo.

Para finalizar, con ello no pretendo realizar una reseña sobre metodología, sino que quiero significar que a pesar de que en las ciencias de la educación existen diferentes formas de referirse a la naturaleza, tipo y diseño de investigación contenidos en todo trabajo, orientado en el paradigma positivista, con enfoque metodológico cuantitativo, mi intención fue ofrecer de manera explicativa a qué se refiere cada uno de los elementos que contienen los aspectos antes mencionados, en el entendido que no son los únicos, pero que traté de presentarlos de la manera más sencilla y clara, atendiendo a mi postura como persona estudiosa interesada en el tema.

\section{Referencias}

Hernández, R., Fernández, C. y Baptista, L. (2010a,b,c). Metodología de la Investigación. México. McGraw-Hill.

Hurtado, I. y Toro, J. (2006). Paradigmas y Métodos de Investigación en Tiempos de Cambio. 5ta Edición. Valencia. Episteme Consultores Asociados C.A.

Kuhn, T. (1981). La Estructura de las Revoluciones Científicas. FCE. México.

Kuhn, T. (1971). Las Estructuras de las Revoluciones Científicas. (Agustín Contin, trad.). México: Fondo de Cultura Económica (obra original publicada en 1962). Recuperada de: 
https://materiainvestigacion.files.wordpress.com/2016/05/kuhn1971.pdf Moreno, A. (2008). El Aro y la Trama: Episteme, Modernidad y Pueblo. Caracas. Editorial Convivium Press, ISBN: 9781934996003.

Ollarves, V. (2015a,b,c,d). Triada Metodológica de la Investigación desde

la Perspectiva Positivista. III Encuentro Nacional para la Socialización del Conocimiento desde el Ser: Un Escenario Académico para la Dialogicidad. Ponencia llevada a cabo en el Programa Postdoctoral en Estudios Libres. UFT, Cabudare, estado Lara, Venezuela.

Ritchey, F.J. (2001). Estadística para las Ciencias Sociales. México, Ediciones: McGraw-Hill.

Rivero, A. (2008). Investigación, creatividad y aprendizaje. Un desafío para el docente en el contexto de la educación venezolana. Tesis doctoral no publicada. Universidad Fermín toro.

UPEL (2008a,b). Reglamento de Estudios de Postgrado. Resolución nro. 2008.316.2018, Extraordinaria nro. 3-2008. Universidad Pedagógica Experimental Libertador. Paraguaná. 


\section{Neolany María Prieto Rivero \\ e-mail: neolany.prieto@gmail.com}

Nacida en Barquisimeto, Estado Lara, Venezuela.

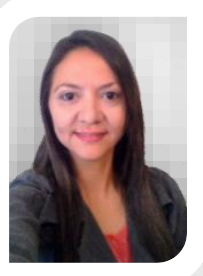
Licenciada en Administración (Universidad Centroccidental Lisandro Alvarado, UCLA), mención Magna Cum Laude. Profesora en Educación Comercial (Universidad Pedagógica Experimental Libertador, Instituto Pedagógico de Barquisimeto). Magíster en Contaduría (UCLA), Magíster en Orientación de la Conducta (Centro de Investigaciones Psicológicas, Psiquiátricas y Sexológicas de Venezuela). Cursante del Doctorado en Ciencias de la Educación (Universidad Fermín Toro). Experto en Educación Virtual en la Fundación para la actualización tecnológica de Latinoamérica (FATLA). Profesor Instructor en la Universidad Pedagógica Experimental Libertador (UPEL-IPB). Inscrita en las líneas de investigación: Estrategias, Recursos e Innovaciones de la Educación Técnica y Tecnologías de Información y Comunicación, Docencia e Innovación. 